\title{
Interventions for Improving Affective Abilities in Adolescents: An Integrative Review Across Community and Clinical Samples of Adolescents
}

\author{
Joyce H. L. Lui ${ }^{1} \cdot$ Christopher T. Barry $^{1} \cdot$ Carmen S. Sergiou $^{2}$
}

Received: 11 October 2016/ Accepted: 27 October 2016/Published online: 7 November 2016

(C) Springer International Publishing 2016

\begin{abstract}
A large body of literature suggests that adolescents with callous-unemotional traits have significant affective impairments, yet few evidence-based interventions exist for this population. The current article reviews the literature on interventions that target emotion recognition and/or perspective-taking abilities among adolescents to identify common processes and features that might help inform further development of treatment strategies for youth with callous-unemotional traits. Thirty-two articles were included and involved samples of typically-developing youth, youth with autism spectrum disorder, youth with antisocial behavior/conduct problems, and youth with disabilities. Overall, interventions for emotion recognition and perspective-taking for adolescents appear promising. Integrating across populations, commonalities among interventions associated with positive outcomes were identified. Recommendations for informing treatment for youth with callous-unemotional traits are discussed.
\end{abstract}

Keywords Emotion recognition $\cdot$ Perspective-taking · Intervention · Adolescents

Joyce H. L. Lui

joyce.lui@wsu.edu

Christopher T. Barry

chris.barry@wsu.edu

Carmen S. Sergiou

cs.sergiou@gmail.com

1 Psychology Department, Washington State University, Johnson Tower 233, PO Box 644820, Pullman, WA 99164-4820, USA

2 Psychology Department, University of Amsterdam, Amsterdam, The Netherlands

\section{Introduction}

Youth with callous-unemotional traits demonstrate significant affective impairments, such as deficits in accurate emotion recognition across various types of emotions in facial, vocal, and body postural cues (Blair et al. 2005; Dadds et al. 2006; Leist and Dadds 2009; Muñoz 2009; Stevens et al. 2001), deficits in perspective-taking (Anastassiou-Hadjicharalambous and Warden 2008; Lui et al. 2016), and deficits in both cognitive and affective empathy (Lui et al. 2016; Muñoz et al. 2011). Furthermore, youth with callous-unemotional traits often follow a particularly pernicious trajectory of severe and persistent conduct problems and antisocial outcomes (Frick et al. 2014). Given the large body of literature documenting deficits and impariments seen among youth with callous-unemotional traits, it is surprising that few, if any, evidence-based treatments exist for this population.

The prevailing impression is that youth with callousunemotional traits do not respond to existing standard treatment. Indeed, a recent review identified a robust association between callous-unemotional traits and poor treatment outcomes among such youth (Hawes et al. 2014). There is now growing consensus that interventions for youth with callous-unemotional traits require adaptations that will address the distinct cognitive and emotional processing deficits and motivational style associated with callousunemotional traits (Frick et al. 2014). Given the affective impairments often seen in such youth, designing interventions that specifically target these areas seems prudent.

Only two known studies to date reported on treatments for youth with callous-unemotional traits that addressed these emotional processing deficits. Dadds et al. (2012) found that training children with high levels of callous-unemotional traits on facial emotion recognition skills in addition to 
providing standard parent training was not associated significant improvement in emotion recognition accuracy, but such youth demonstrated greater reduction of conduct problems compared to those who did not receive the emotion recognition training. In a case study, Datyner et al. (2016) found that an emotional training intervention resulted in improved empathic responding in a 7-year-old child with callous-unemotional traits. However, the case study design precludes conclusions regarding whether such intervention may be efficacious with other children. Across these two studies, findings are mixed, at least regarding primary outcomes. Furthermore, these studies involved younger samples, and it remains unknown if similar interventions may be appropriate for an older adolescent population.

\section{Current Review}

Because no evidence-based interventions to address affective deficits for adolescents with callous-unemotional traits currently exist, this review draws on literature across different target populations to identify interventions that are effective at improving emotion recognition and perspectivetaking among adolescents. The review focuses on interventions that either exclusively target emotion recognition or perspective-taking abilities or address these skills within a broader treatment program (e.g., social skills training). Emotion recognition is defined as the identification of others' emotions from perceptual or sensory cues (i.e., face, voice, body posture), whereas perspective-taking is defined as the identification or inference of others' emotions based on their context or situations. The goal of the review is to identify common processes, themes, and components across these interventions that can inform further development of treatment strategies for adolescents with callous-unemotional traits. To the extent that common features exist among efficacious interventions, a transdiagnostic approach to targeting these affective abilities in adolescents may be fruitful. Thus, the current review examined the following questions: (1) what is the current empirical basis for interventions targeting perspective-taking and emotion recognition abilities, (2) what are common features among interventions that are efficacious or that show promising results, and (3) how can existing studies inform new or refine interventions for youth with callous-unemotional traits?

\section{Method}

\section{Study Selection}

A literature search was conducted using PsycINFO with inclusion criteria specified as peer-reviewed, in the English language, and restricted to the 6-12 and 13-17 year-old age categories in PsycINFO. Search terms included: (emotion recognition OR empathy OR perspective taking) AND (training OR intervention OR teaching). An empathy search term was included to capture studies assessing perspective-taking but that were framed as cognitive empathy by researchers. Articles were then screened at the title and abstract level. This approach resulted in 195 full text articles for detailed examination for inclusion in the current review. Articles were excluded if (1) no outcome data were reported $(k=17)$, (2) participants were outside the age range of 10-17 years of age since the population of interest is adolescents $(k=91){ }^{1}$ (3) the research design involved single case or multiple baseline designs $(k=10)$, (4) the intervention did not target perspective-taking or emotion recognition in whole or in part $(k=29)$, and (5) perspective-taking or emotion recognition was not assessed as an outcome $(k=10)^{2}$ Reviews and meta-analyses $(k=8)$ were examined for the relevant primary articles. The reference lists of articles were also examined for relevant literature. Two additional articles were identified from references. This approach resulted in a final sample of 32 articles in the current review.

\section{Study Coding}

Information regarding the demographics of the sample, treatment design, and treatment outcome were coded for each article. Demographic variables included sample size, type of sample, gender, ethnicity, age, socioeconomic status, and intelligence of the participants. Treatment design variables included specificity of the intervention, stimuli and procedure of the intervention, format/delivery, intensity, setting, leader, parental involvement, and assignment into group(s). Treatment outcome variables included outcome measure, follow-up data, treatment success, and moderators.

\footnotetext{
1 Articles that included samples outside the age range of 10-17 years were inspected on a case-by-case basis. If the reported mean age of the sample was 10 years or above, it was included in the review. One study (Blancher-Dixon and Simeonsson 1978) involved a sample of adolescents with intellectual disability. Although the mean chronological age for this sample was 10.3 years, the authors noted that the mental age of the participants ranged from 5 to 9 years. Thus, this study was excluded in the current review.

${ }^{2}$ Studies that broadly targeted social skills or theory of mind were excluded unless the researchers specified a component that addressed perspective-taking or emotion recognition and also measured it as an outcome. The majority of studies that were excluded were based on empathy interventions.
} 


\section{Results}

\section{Interventions with Community Samples}

Of the 32 articles included in this review, 11 involved community samples. The remaining 21 involved samples of youth with Autism Spectrum Disorder, antisocial/ delinquent behaviors, learning impairment, or visual or auditory impairment. All but one of the studies with community samples involved an intervention that targeted perspective-taking skills (Bridgeman 1981; Bulkeley and Cramer 1994; Castillo et al. 2013; Lane-Garon 1998; Marsh et al. 1980; Nakkula and Nikitopoulos 2001; Schonert-Reichl et al. 2012, 2015; Silvern et al. 1979; Wölfer et al. 2014). Only Emerson and Holden's (1991) study involved an emotion recognition skills intervention with a community sample.

Demographic variables across the community samples are presented in Table 1. Overall, there was roughly equal representation of males and females across studies $\left(M_{f e}\right.$ male $\left.=49.4 \%, M_{\text {male }}=50.7 \%\right)$. Participants were predominantly early adolescents, around ages 10-13 years. Many studies did not report on the ethnicity $(45.5 \%)$ or the socioeconomic status $(63.6 \%)$ of the sample. From the available data, participants were mostly middle-class Caucasian/White/native English speakers.

Perspective-taking interventions varied in their specificity. Four studies focused specifically on perspectivetaking skills interventions (i.e., Bridgeman 1981; LaneGaron 1998; Silvern et al. 1979; Marsh et al. 1980), one focused on cyberbullying prevention (Wölfer et al. 2014), one focused on negotiation training (Nakkula and Nikitopoulos 2001), and four focused on larger social-emotional learning or social skills training (i.e., Bulkeley and Cramer 1994; Castillo et al. 2013; Schonert-Reichl et al. 2012, 2015). A description of intervention characteristics across studies is presented in Table 2. All studies employed a group intervention implemented within a school setting. Parents were typically not involved in the intervention beyond providing data on youth behavior at pre- and posttreatment. The interventions were predominantly led by teachers, except for Bukleley and Cramer's (1994) and Castillo et al.'s (2013) interventions, which were led by therapists and psychologists involved in the research. Interventions also varied substantially in intensity, ranging from six 40-min sessions to weekly hour-long sessions spanning 2 years. The majority of studies $(60 \%)$ involved some form of randomization. Three studies randomly assigned students across treatment conditions (Bulkeley and Cramer 1994; Schonert-Reichl et al. 2015; Silvern et al. 1979), and three studies randomly assigned classrooms across treatment conditions, as the interventions involved were class- or school-wide (Bridgeman 1981; Castillo et al. 2013; Wölfer et al. 2014). Treatment groups were commonly compared to treatment as usual, which predominantly involved typical class/school activities. Materials and procedures used in perspective-taking training were also heterogeneous across studies. The majority of studies $(77.8 \%)$ implemented a manualized or structured program (e.g., INTEMO in Castillo et al. 2013; MindUP in Schonert-Reichl et al. 2015), whereas others were semistructured involving scripts or videotaped skits followed by open discussions (e.g., Bridgeman 1981; Marsh et al. 1980; Silvern et al. 1979). Interventions frequently involved group discussions among peers (70\%). Many interventions (80\%) also included experiential activities, such as roleplays of pre-assigned and/or relevant personal social situations, videotaping and playbacks of role-plays as a way to initiate and generate discussions or to provide feedback (e.g., Marsh et al. 1980), reflective activities such as creating art projects and films based on content of the training sessions (e.g., Castillo et al. 2013), and homework of "real life assignments" to practice implementing skills learned in sessions (e.g., Bulkeley and Cramer 1994). Half of the studies also included modeling of skills by a peer or adult either via video or live demonstrations (e.g., Bridgeman 1981). A smaller number of studies (40\%) involved modeling of skills (e.g., Bridgeman 1981) and explicit instructions of skills (e.g., Silvern et al. 1979).

Of the ten interventions that targeted perspective-taking skills, seven demonstrated positive outcomes, with an average medium effect size (e.g., Bridgeman 1981; Bulkeley and Cramer 1994; Lane-Garon 1998; Nakkula and Nikitopoulos 2001; Schonert-Reichl et al. 2015; Silvern et al. 1979; Wölfer et al. 2014), one demonstrated a partial positive outcome (i.e., Castillo et al. 2013), and two did not demonstrate significant improvements as a result of treatment (i.e., Marsh et al. 1980; Schonert-Reichl et al. 2012). Perspective-taking was typically assessed via questionnaires, predominantly $(50 \%)$ by the Perspective Taking subscale of Davis's Interpersonal Reactivity Index (1983). Other researchers employed task-based assessments of perspective-taking skills, such as presenting the participant with vignettes, pictures, cartoons, or social scenes and asking the participant to identify the character's emotions and thoughts or providing the participant with privileged information and subsequently asking the participant to explain or retell a scenario from an unknown person's point-of-view. None of these studies collected follow-up data or explored moderator effects.

The single emotion recognition intervention (Emerson and Holden 1991) focused exclusively on improving this skill. It similarly involved a group intervention conducted in a school setting for fifth graders. Emerson and Holden 


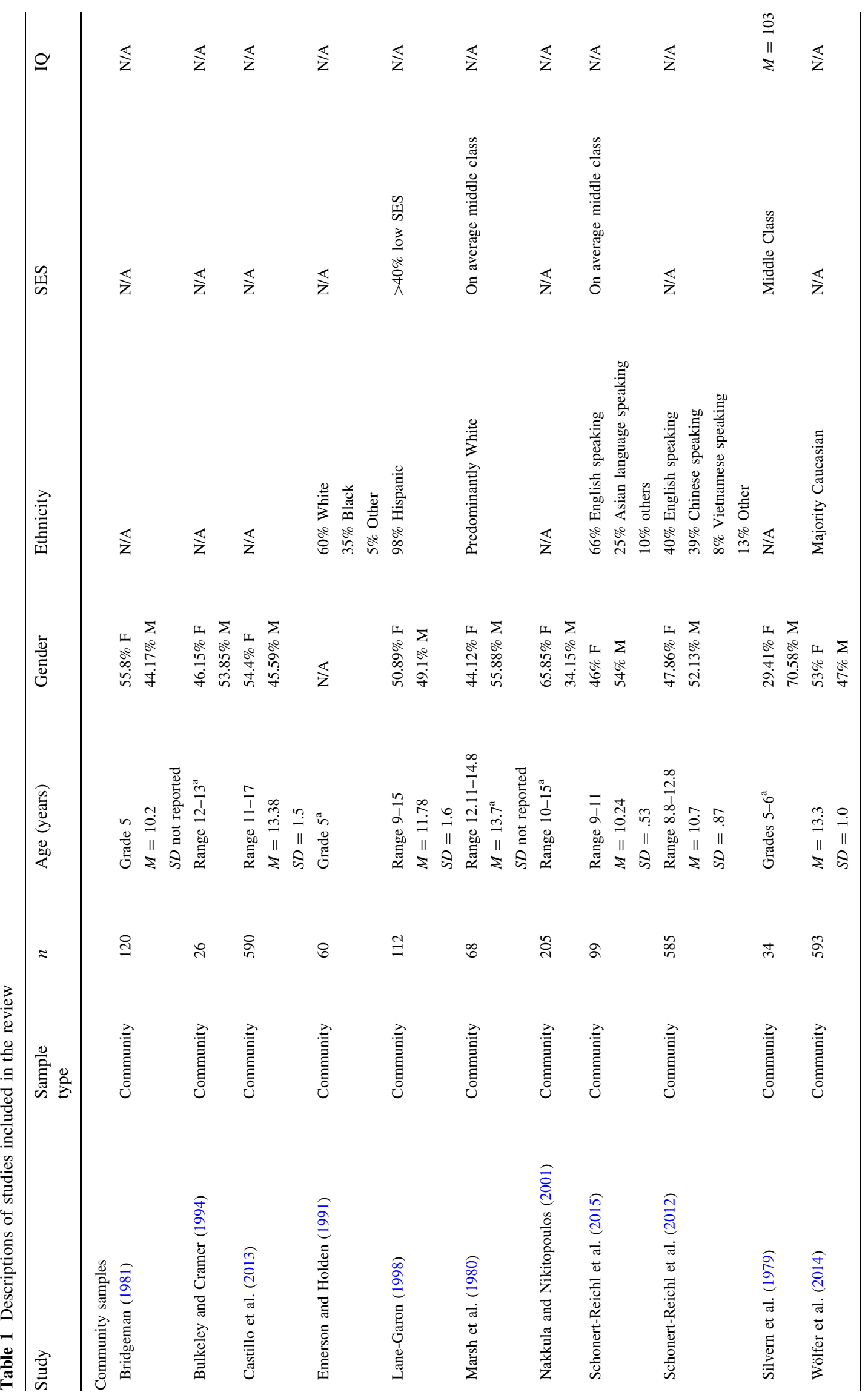




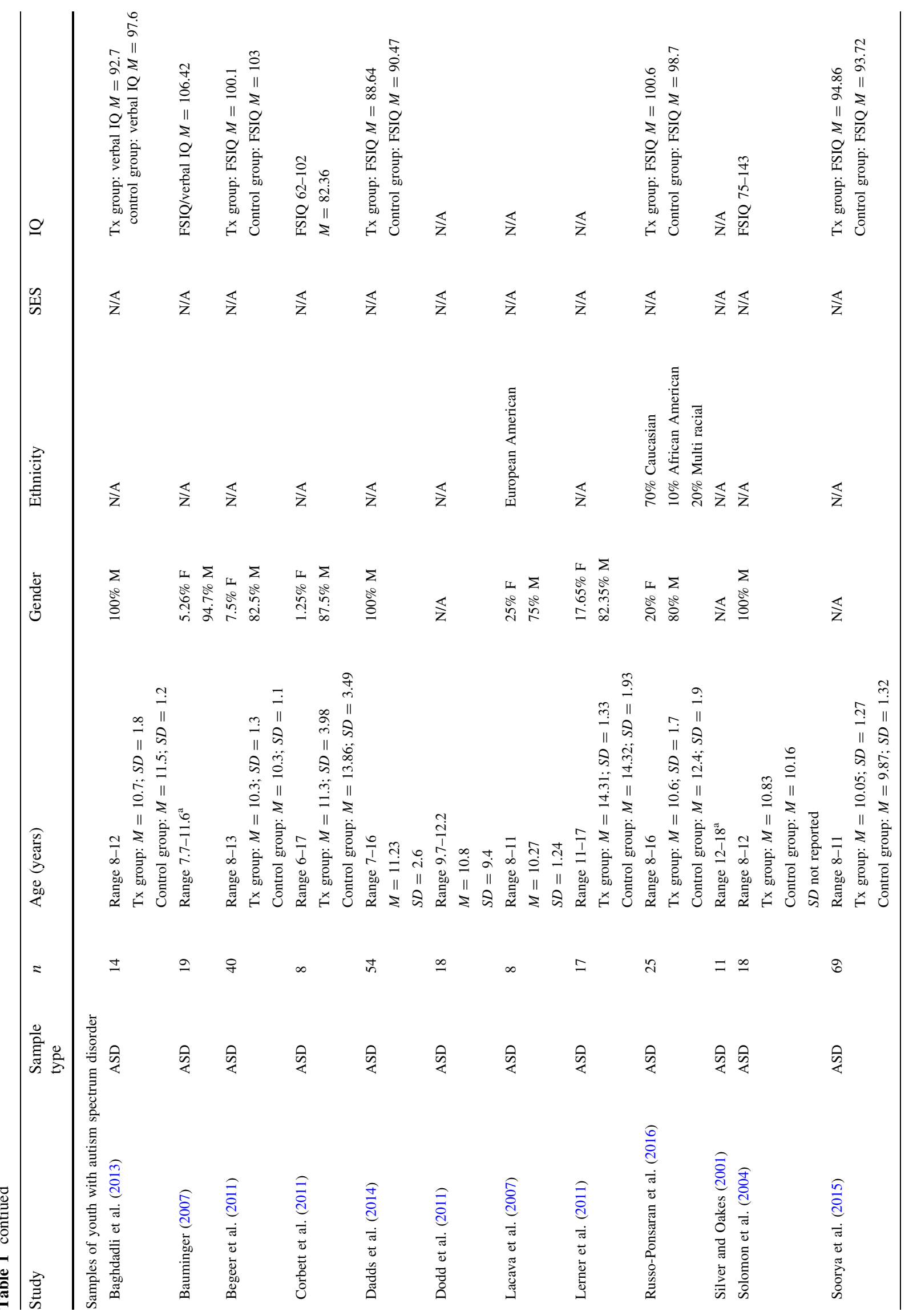




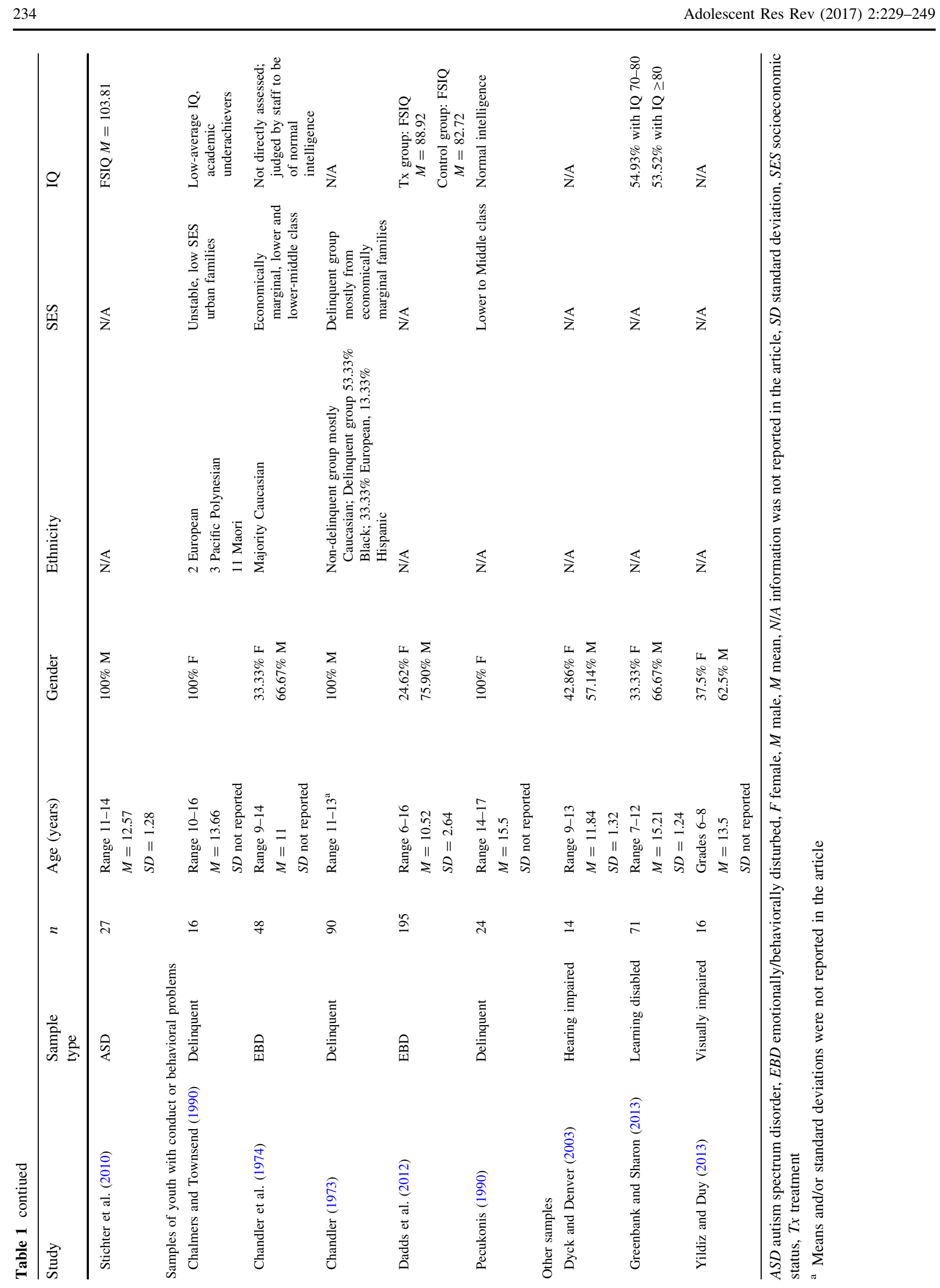

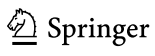




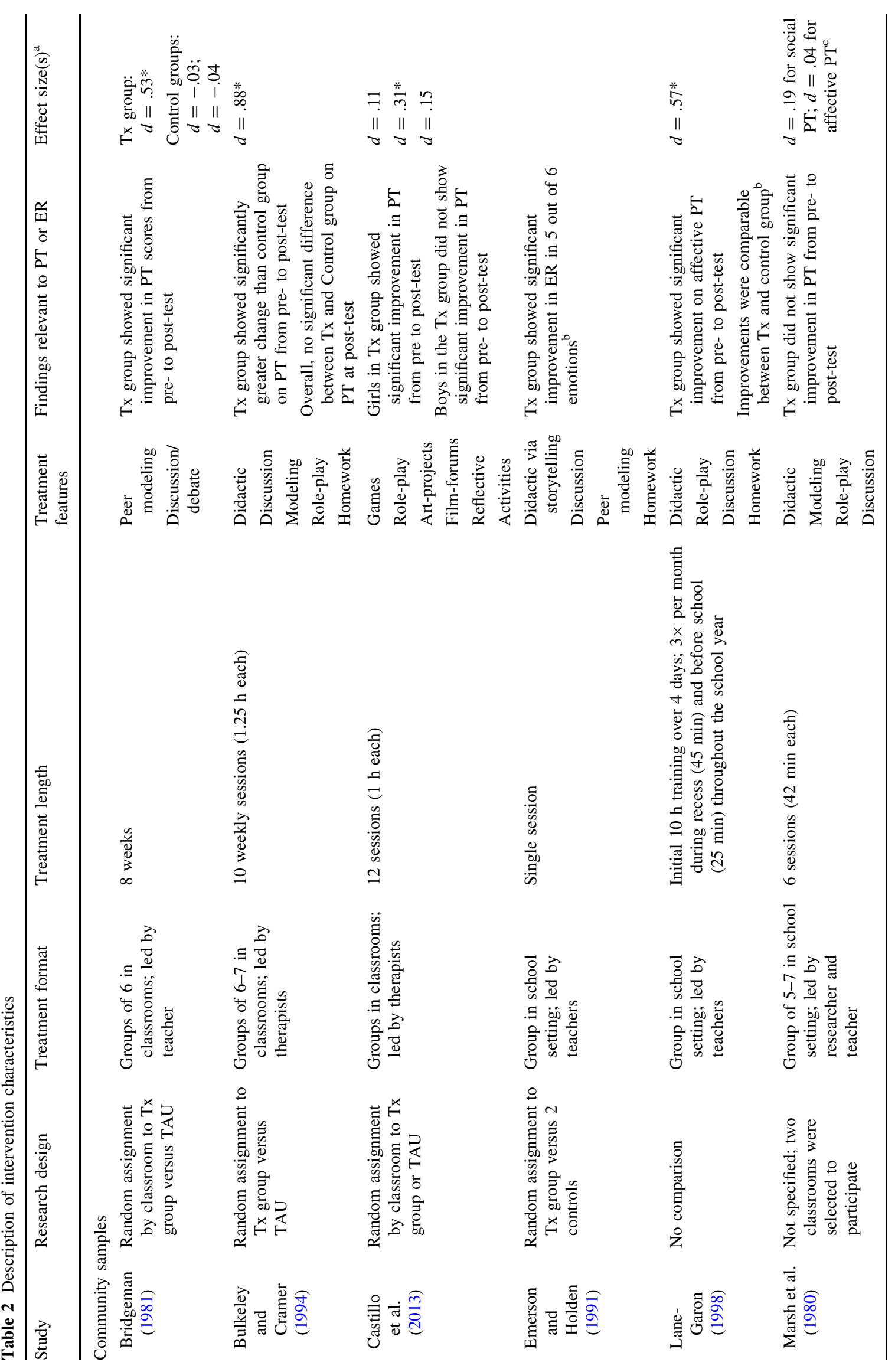




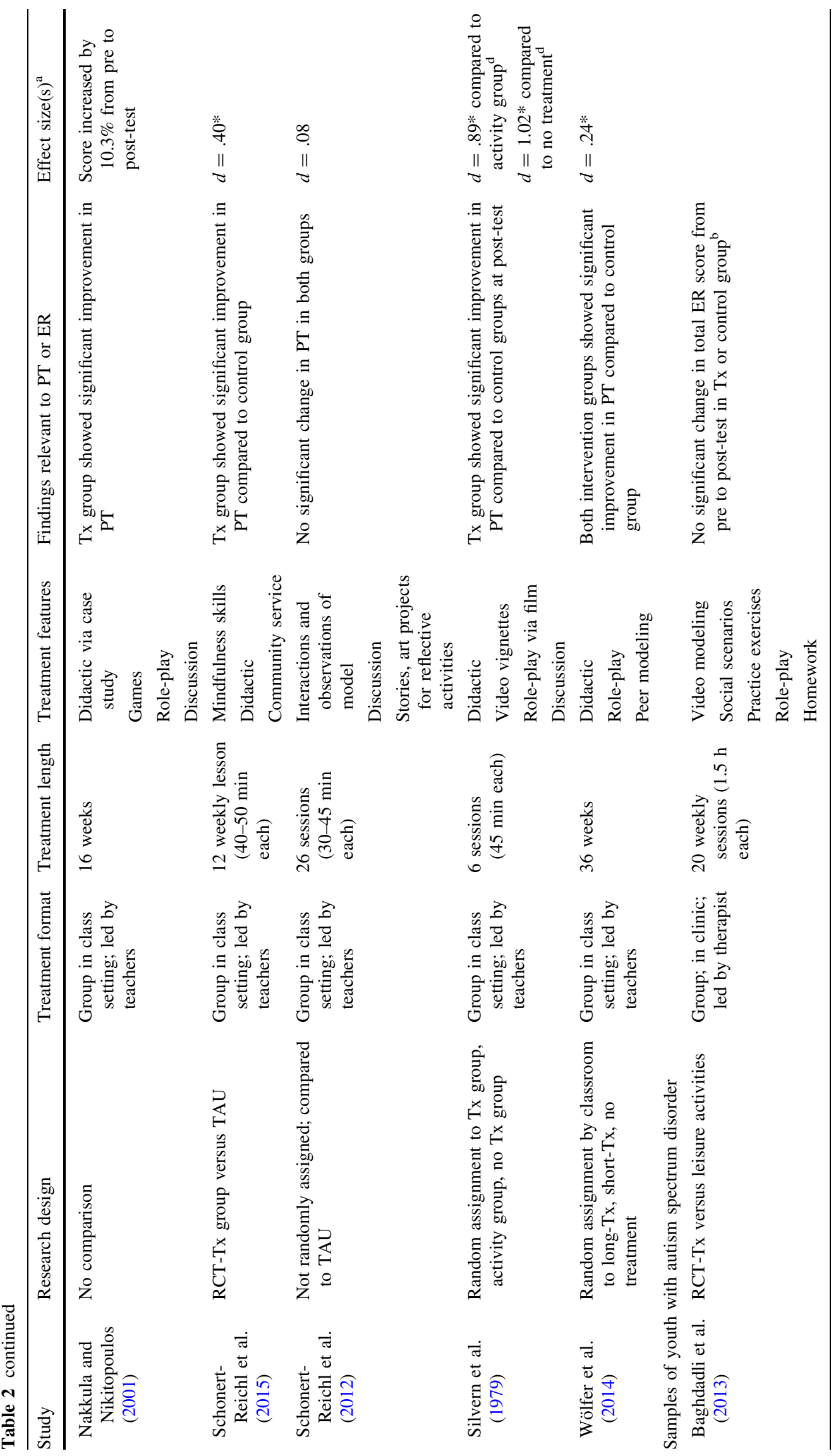




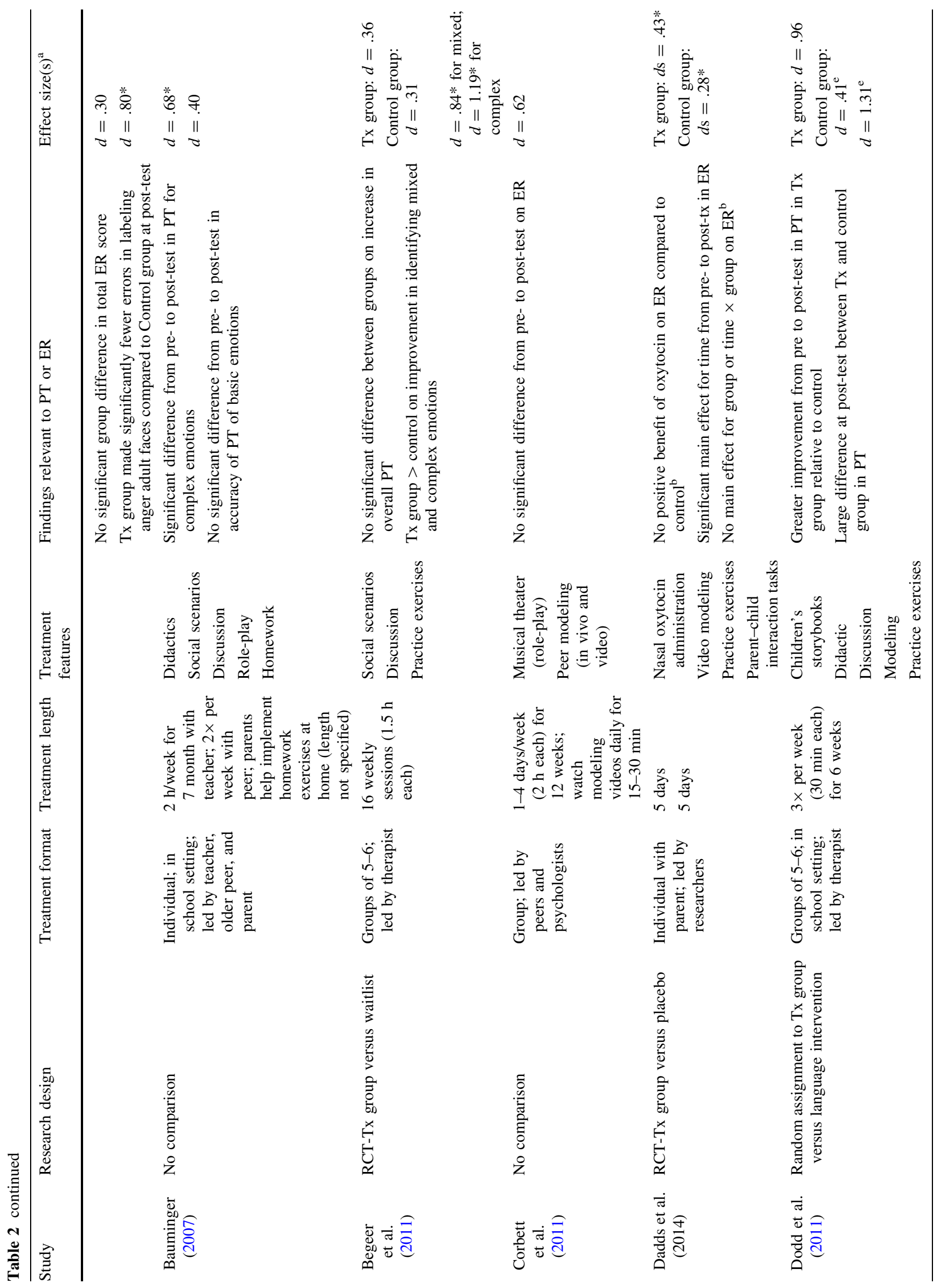




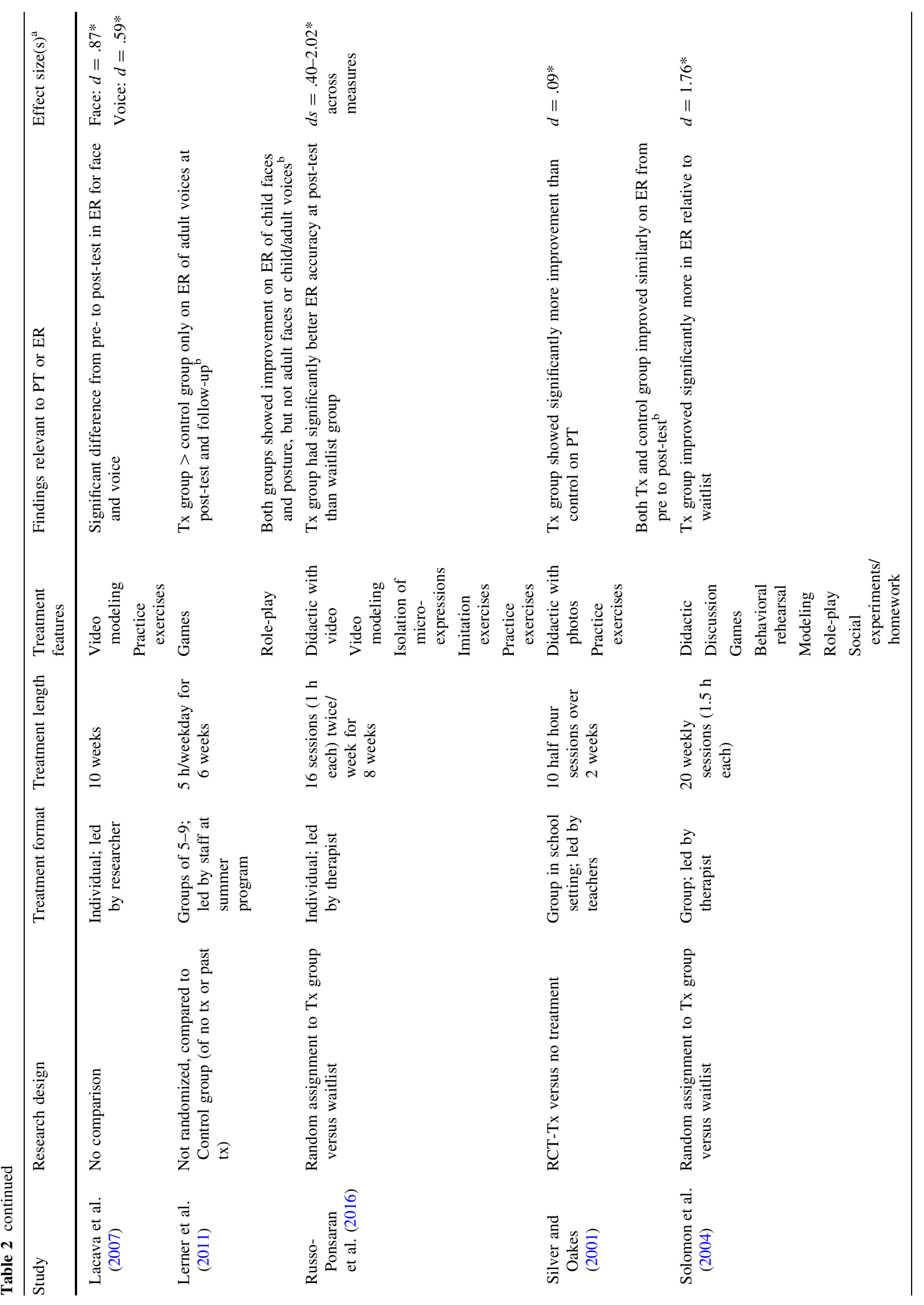




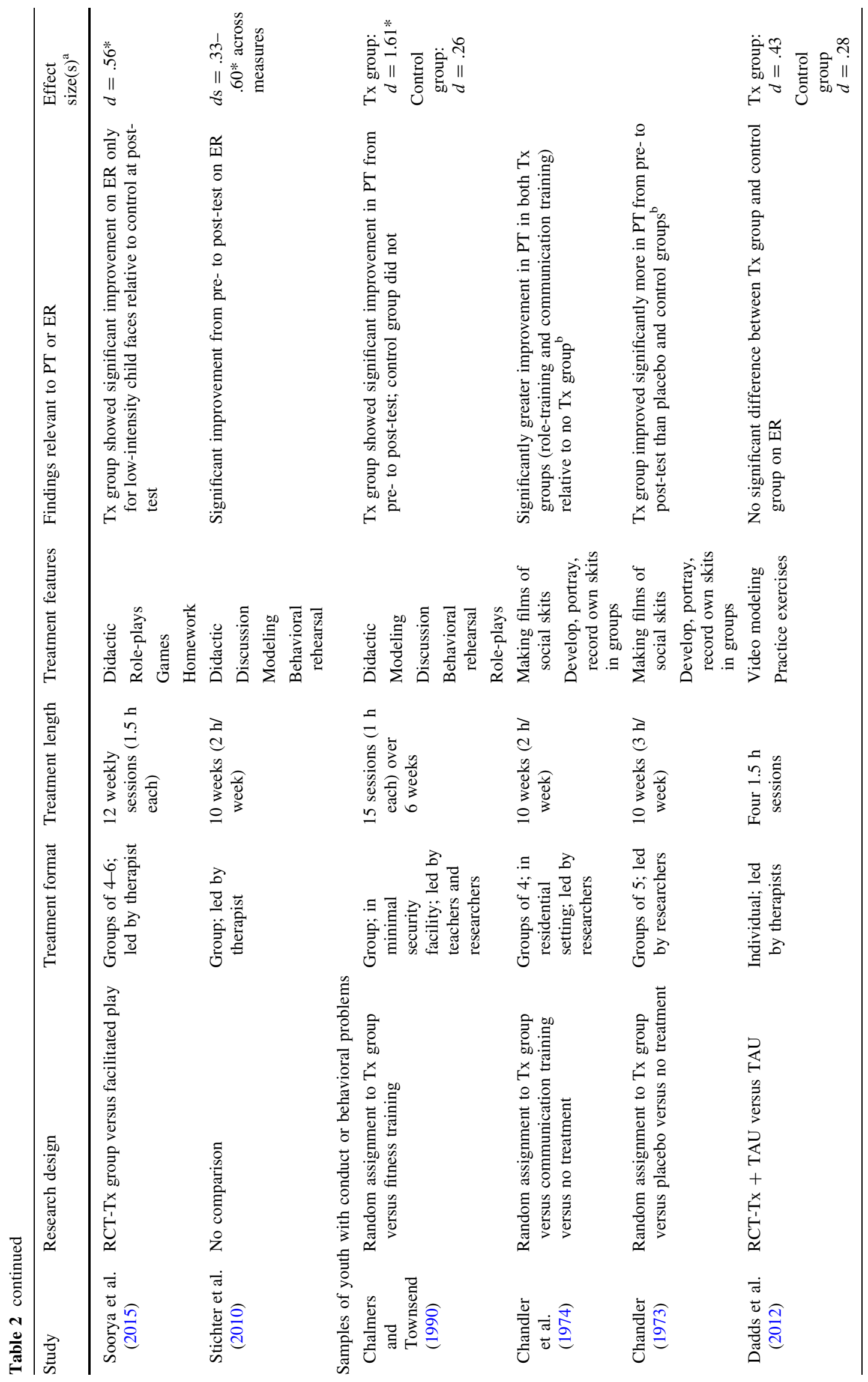




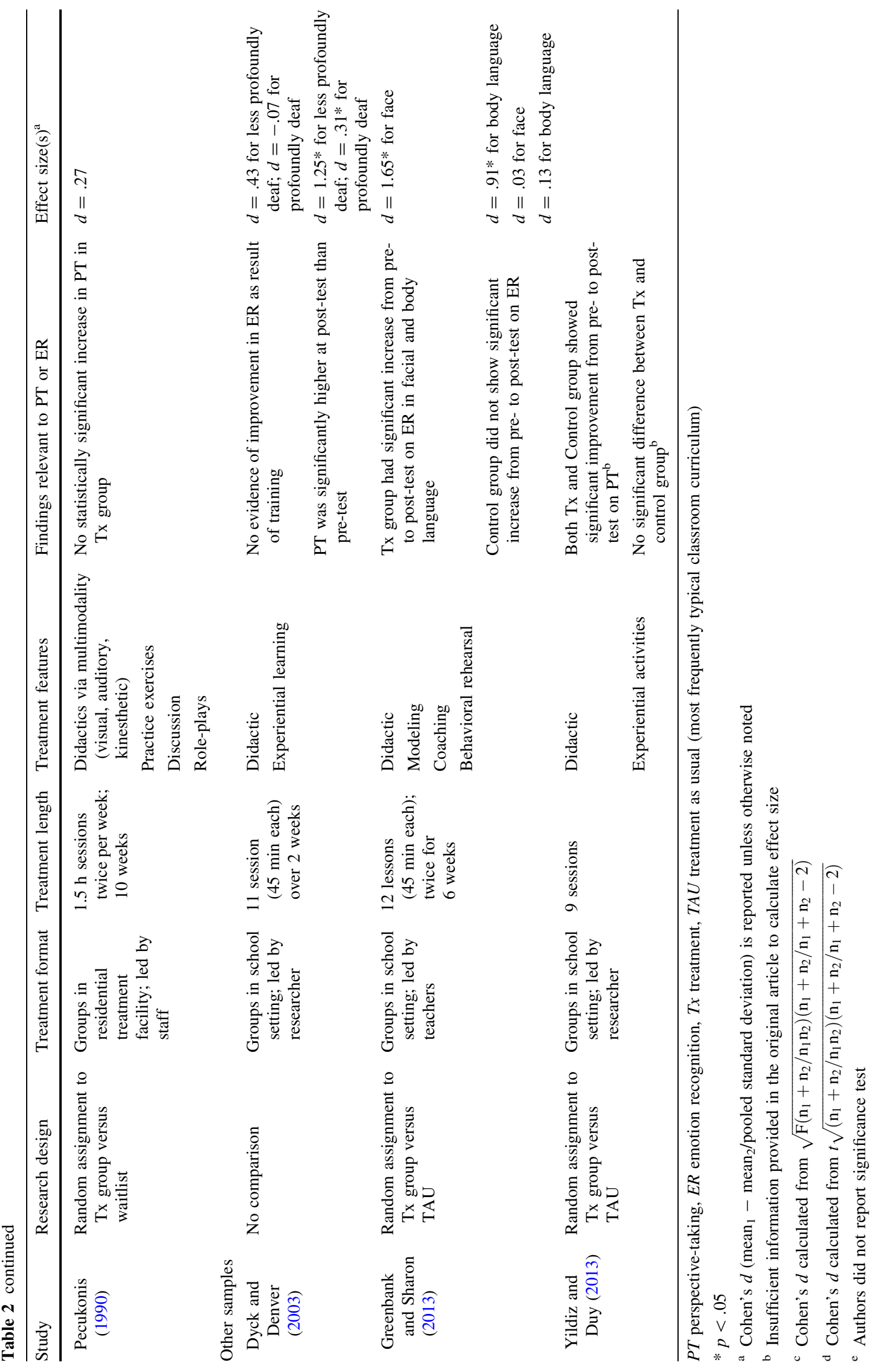


(1991) randomly assigned participants across treatment and no treatment conditions. The researchers employed a narrative and discussion-based intervention that lasted a single session. Participants were read a story depicting a cartoon protagonist experiencing six emotions (i.e., sad, tired, afraid, angry, unsure, and happy) and physical cues corresponding to each (e.g., "Rusty began to feel very happy. His mouth curled up on the ends into a big smile."). Participants in the group were asked questions about each emotion in the story followed by a discussion of how the participants themselves experience each emotion and the physical signs associated with them. Furthermore, pictures were taken of peers (not in the study) modeling the emotions. Participants practiced identifying the emotions in the pictures as well as with other peers throughout the day. After the training, all groups completed an emotion recognition task that probed for how an emotion feels (e.g., "how does sad feel?"). Responses were coded on a 0-5 scale by trained teachers. Results indicated that the treatment group significantly improved their post-treatment scores relative to their pre-treatment scores for all six emotions, whereas there were no significant differences between pre-and post-treatment scores in the no treatment conditions.

\section{Interventions with Individuals with Autism Spectrum Disorder}

The largest group of articles (13) identified in this review involved samples of youth with autism-spectrum disorder (40.6\%). The majority (69.2\%) of the studies with samples of individuals diagnosed with autism-spectrum disorder involved an intervention that targeted emotion recognition skills (Baghdadli et al. 2013; Corbett et al. 2011; Dadds et al. 2014; Lacava et al. 2007; Lerner et al. 2011; RussoPonsaran et al. 2016; Stichter et al. 2010; Solomon et al. 2004; Soorya et al. 2015), whereas $23.1 \%$ involved an intervention that targeted perspective-taking skills (Bauminger 2007; Begeer et al. 2011; Dodd et al. 2011). One study involved an intervention that targeted both skills (Silver and Oakes 2001).

Demographic variables are presented in Table 1. Overall, males are overrepresented in these studies $\left(M_{f e}\right.$ male $=7.7 \%, M_{\text {male }}=90.2 \%$ ). Although the prevalence rate of autism-spectrum disorder is higher among males than females, the gender ratio across studies is more imbalanced than is typically reported (e.g., four times more likely in males than females; American Psychiatric Association 2013). Participants were early to middle adolescents, around ages of 10-14 years. Only two studies reported on ethnicity (predominately Caucasian), and none reported on the socioeconomic status of the sample. All studies had inclusion criteria specifying a formal current or past diagnosis of Autism Spectrum Disorder (e.g., autism, Asperger syndrome, and pervasive developmental disordernot otherwise specified) based on diagnostic criteria from the Diagnostic and Statistical Manual of Mental DisordersIV or International Statistical Classification of Diseases and Related Health Problems-10. Some studies corroborated the diagnosis by assessment with the Autism Diagnostic Observation Schedule and Autism Diagnostic Interview-Revised (e.g., Baghdadli et al. 2013; Soorya et al. 2015). All studies excluded participants with intellectual disabilities, established via a formal assessment with standardized instruments (e.g., Wechsler-Intelligence Scale for Children-IV). Nine studies reported on the intellectual functioning of the sample, with the majority of samples having a mean Full Scale Intelligence Quotient within the normative range (i.e., 85-115).

Emotion recognition interventions varied in their specificity. The majority of studies (70\%) included emotion recognition as a component of a larger social skills training package (Baghdadli et al. 2013; Corbett et al. 2011; Dadds et al. 2014; Lerner et al. 2011; Stichter et al. 2010; Soorya et al. 2015; Solomon et al. 2004). Three specifically focused on emotion recognition or emotional understanding (Lacava et al. 2007; Russo-Ponsaran et al. 2016; Silver and Oakes 2001). A description of intervention characteristics across studies is presented in Table 2. Seven of the studies that targeted emotion recognition skills were groupbased and were predominantly led by a therapist, psychologist, or researcher. Four studies involved parents within the interventions, whereas six studies had no, or minimal, parental involvement beyond providing assessment data. Interventions with parental involvement included a parallel psychoeducational group (Solomon et al. 2004; Soorya et al. 2015; Stichter et al. 2010), with the exception of the Dadds et al. (2014) study in which parents were included in parent-child training sessions concurrently with their children. Interventions varied substantially in duration, ranging from 1 to 20 weeks. Some interventions involved weekly 90 min sessions (e.g., Solomon et al. 2004), and some were more intensive and involved sessions multiple times a week (e.g., Lerner et al. 2011). The majority of studies $(60 \%)$ involved random assignment to treatment conditions. Half of these studies compared the treatment condition to no treatment, and the other half compared the treatment condition to a placebo/active control (e.g., Soorya et al. 2015). Materials and procedures used in emotion recognition training were heterogeneous across studies. Some common features among interventions include didactic of a range of emotions with visual and/or auditory aids (50\%), modeling of emotional expression with the use of video, audio, or peer $(70 \%)$ roleplays or imitation exercises of emotions (60\%), and repeated practice or behavioral reversal of emotion 
identification in sessions and/or outside of sessions (80\%). Some studies involved computerized programs to train emotion recognition (e.g., Mindreading program in Lacava et al. 2007; Dadds et al. 2014; the MiX program in RussoPonsaran et al. 2016; and the Emotion Trainer in Silver and Oakes 2001). The majority of studies addressed recognition of facial expressions exclusively (Silver and Oakes 2001), whereas others also targeted emotion recognition from auditory and postural cues (e.g., Lerner et al. 2011).

Of the ten interventions that targeted emotion recognition, four reported positive outcomes (Lacava et al. 2007; Russo-Ponsaran et al. 2016; Stichter et al. 2010; Solomon et al. 2004). Participants in these four studies demonstrated significant improvements in emotion recognition from preto post-treatment, with an average medium effect size, and significant improvement relative to waitlist conditions, with large effect sizes. Five studies demonstrated mixed outcomes (Baghdadli et al. 2013; Dadds et al. 2014; Lerner et al. 2011; Silver and Oakes 2001; Soorya et al. 2015). Most of these studies demonstrated positive findings for only one of several outcome indices, such as greater emotion recognition for adult angry faces (Baghdadli et al. 2013), adult voices (Lerner et al. 2011), and low-intensity child faces (Soorya et al. 2015), but they generally did not find significant differences between the treatment and control conditions. One study (Corbett et al. 2011) did not find significant improvement in emotion recognition as a result of the intervention. Emotion recognition was assessed by tasks, most frequently by the Diagnostic Analysis of Non-verbal Accuracy-2, in which participants are presented with an emotional stimulus (e.g., facial expression), and they are asked to identify the emotion depicted from a list of response options. Only three studies collected follow-up data (within 1-3 months post-treatment), and results remained similar in these studies. Only one study (Soorya et al. 2015) explored moderator effects, although no significant moderation involved emotion recognition indices.

Four studies (three exclusively targeting perspectivetaking and one targeting both emotion recognition and perspective-taking) reported on perspective-taking interventions (Bauminger 2007; Begeer et al. 2011; Dodd et al. 2011; Silver and Oakes 2001). Three of these studies targeted perspective-taking as part of a larger social skills or social emotion intervention, and one specifically addressed emotional understanding. A description of intervention characteristics across studies is presented in Table 2. Three of the studies (Begeer et al. 2011; Dodd et al. 2011; Silver and Oakes 2001) were group-based, and three of the studies were (Bauminger 2007; Dodd et al. 2011; Silver and Oakes 2001) implemented in a school setting. There were equal numbers of interventions led by teachers versus therapists. Only one study (Bauminger 2007) did not involve random assignment. The rest of the studies randomly assigned participants to a treatment versus a waitlist/no treatment or active control group. Interventions across the studies varied in intensity, ranging from 30-min sessions every 2 weeks to weekly 2 -h sessions for 28 weeks. In two studies, parents were not involved beyond providing assessment data. In the other two studies, parents were provided psychoeducation sessions (Begeer et al. 2011) and were actively involved in implementing the practice of skills between sessions with their child (Bauminger 2007).

All four perspective-taking interventions involved some form of practice exercises, either by retelling stories from another's perspective (Dodd et al. 2011), identifying perspectives in hypothetical situations (Begeer et al. 2011; Silver and Oakes 2001), or engaging in role-plays (Bauminger 2007). Three of the studies also involved discussions. One study demonstrated positive outcomes, as Dodd et al. (2011) found that participants in the treatment group showed greater improvement in perspective-taking relative to the control group, with a large effect size. Three studies demonstrated mixed outcomes (Bauminger 2007; Begeer et al. 2011; Silver and Oakes 2001). Bauminger (2007) found that the treatment group demonstrated significant improvement in understanding the perspective of complex emotions but not of basic emotions. Begeer et al. (2011) found that there was comparable improvement across the treatment and control groups in perspective-taking, except for with mixed and complex emotions, where the treatment group improved significantly more. Finally, Silver and Oakes (2001) found that both the treatment and control groups improved comparably on perspective-taking. All of the outcome assessments involved vignettes, and participants were asked to identify the emotions that the target in the vignette was experiencing and to justify their responses. None of the studies collected follow-up data or examined moderators.

\section{Interventions with Youth Experiencing Conduct or Behavioral Problems}

Five articles involved samples with conduct or behavioral issues (Chalmers and Townsend 1990; Chandler 1973; Chandler et al. 1974; Dadds et al. 2012; Pecukonis 1990). All but one of the studies involved an intervention for perspective-taking skills. Only the Dadds et al. (2012) study involved an intervention for emotion recognition.

Demographic variables across the samples involving youth with conduct problems are presented in Table 1 . Overall, there was slightly more representation of females than males across studies $\left(M_{\text {female }}=58.3 \%\right.$, $M_{\text {male }}=41.8 \%$ ), contrary to prevalence rates of conduct or behavioral problems, which is typically higher among males (American Psychiatric Association 2013). 
Participants were predominantly early adolescents, although they ranged in age from 6 to 17 years. Only three studies reported on the ethnicity of participants (Chalmers and Townsend 1990; Chandler 1973; Chandler et al. 1974), and ethnicity varied widely across these samples. For example, in Chalmers and Townsend's (1990) study, the majority of participants were of indigenous descent, whereas in Chandler et al.'s (1974) study, the majority of participants were White/Caucasian. Four of the studies reported participants' socioeconomic statuses, and participants on average were from low income or marginal economic backgrounds. Participants in these studies were selected based on police or court contact (Chandler 1973; Pecukonis 1990), or they were otherwise detained, referred, or in treatment for behavioral problems (Chalmers and Townsend 1990; Chandler et al. 1974; Dadds et al. 2012).

Three studies specifically targeted perspective-taking as the point of intervention (Chalmers and Townsend 1990; Chandler 1973; Chandler et al. 1974), and one study targeted empathy (Pecukonis 1990). A description of intervention characteristics is presented in Table 2. All four studies employed a group intervention and were predominantly led by researchers. Parents were not involved in the intervention in these studies. Three of the interventions lasted 10 weeks, with 2-3 h of intervention per week (Chandler 1973; Chandler et al. 1974; Pecukonis 1990). Chalmers and Townsend's (1990) intervention was shorter term but more intensive (i.e., 15 hourly sessions over 6 weeks). All four studies involved random assignment to treatment versus no treatment, placebo/control, or both. All studies involved some form of role-play and behavioral rehearsal to practice taking on the perspectives of others. Two studies also included peer discussion as a way to provide feedback (e.g., Chalmers and Townsend 1990).

Of the four interventions that targeted perspective-taking skills for youth with behavioral problems, two demonstrated positive outcomes (Chalmers and Townsend 1990; Chandler 1973), and one demonstrated partial positive outcomes (Chandler et al. 1974). In the first two studies, the treatment group showed significantly greater improvement in perspective-taking relative to the control group. Chandler et al. (1974) similarly found that the treatment group showed significantly greater improvement in perspective-taking than the no treatment group; however, the treatment group performed comparably to an active control group. On the other hand, Pecukonis (1990) did not find statistically significant improvement in perspective-taking in the treatment group as a result of empathy training. The three studies that found at least partial positive results assessed perspective-taking with a performance-based measure (i.e., Chandler's social perspective-taking task), whereas Pecukonis assessed perspective-taking with a self-report questionnaire. No studies collected follow-up data on the outcome of interest or explored moderator effects.

The single emotion recognition intervention (Dadds et al. 2012) focused exclusively on improving this skill. The intervention was individual-based and was led by therapists. The intervention was relatively short, involving four 90-min sessions. Participants were randomly assigned to receive emotion recognition training in addition to treatment as usual or to only receive treatment as usual. Dadds et al. (2012) employed a video interactive training program (Baron-Cohen's Mindreading program) to improve emotion recognition skills. Participants were presented with video models of emotions and engaged in practice exercises of emotion identification. The outcome was assessed via a performance-based measure. Dadds et al. (2012) found that the treatment group did not significantly differ from the treatment as usual group on emotion recognition accuracy after the intervention.

\section{Interventions with Other Samples}

The three remaining articles each targeted a different population. Greenbank and Sharon (2013) examined an emotion recognition intervention among a sample of adolescents with learning disability. Dyck and Denver (2003) examined an intervention targeting both emotion recognition and perspective-taking among adolescents who were hearing impaired. Yildiz and Duy (2013) examined a perspectivetaking intervention among adolescents who were visually impaired. Demographic variables for these studies are presented in Table 1. Overall, males are overrepresented across these studies $\left(M_{\text {female }}=37.9 \%, M_{\text {male }}=62.1 \%\right)$, although the gender ratio is generally consistent with the prevalence rates of disabilities across gender in adolescence, with the exception of visual impairment where prevalence rates are comparable across gender (American Psychiatric Association 2013; U.S. Census Bureau 2015). Participants were again predominantly early adolescents, with an average age of 13.5 years. None of the studies reported on the ethnicity or socioeconomic status of the samples. About half of the sample in Greenback and Sharon's (2013) sample had an intelligence quotient in the range of 70-80.

A description of intervention characteristics is presented in Table 2. The two studies (Dyck and Denver 2003; Yildiz and Duy 2013) that addressed perspective-taking skills were both group-based, implemented in a school setting, and led by researchers. Both interventions were relatively short (9-11 sessions). Parents were not involved in these interventions. Dyck and Denver's (2003) study did not have a comparison group, whereas Yildiz and Duy (2013) randomly assigned participants to treatment versus treatment as usual. Both studies included didactic and experiential activities. Dyck and Denver (2003) found that 
participants demonstrated significant improvement in perspective-taking as a result of training. In contrast, Yildiz and Duy (2013) found that the treatment group improved in perspective-taking comparably to the treatment as usual group.

The studies (Dyck and Denver 2003; Greenbank and Sharon 2013) that addressed emotion recognition were also group-based, implemented in a school setting, and led by researchers. Both interventions involved 11-12 sessions, although Dyck and Denver's (2003) intervention was shorter (i.e., 2 vs. 6 weeks) in span relative to Greenbank and Sharon's (2013) approach. Parents were not involved in these interventions. Greenbank and Sharon (2013) randomly assigned participants to treatment versus treatment as usual. Their intervention involved more modeling and behavioral rehearsal than did the intervention described by Dyck and Denver (2003). Greenbank and Sharon (2013) found that the treatment group showed significantly greater improvement in emotion recognition relative to the control group, whereas Dyck and Denver (2003) did not find evidence of improvement in emotion recognition as a result of training.

\section{Integration Across Populations}

\section{Perspective-Taking}

Across types of populations, the twelve interventions that demonstrated positive effects for perspective-taking had several commonalities (Bauminger 2007; Bridgeman 1981; Bulkeley and Cramer 1994; Chandler 1973; Chandler et al. 1974; Chalmers and Townsend 1990; Dodd et al. 2011; Lane-Garon 1998; Nakkula and Nikitopoulos 2001; Schonert-Reichl et al. 2015; Silvern et al. 1979; Wölfer et al. 2014). About half of them focused exclusively on perspective-taking (Bridgeman 1981; Chandler 1973; Chandler et al. 1974; Chalmers and Townsend 1990; Silvern et al. 1979). All but one intervention were groupbased and implemented in a school or residential/institutional facility. It is possible that group-based interventions may be particularly conducive to learning perspectivetaking skills, because cooperation and interdependence are inherent within a group process, and they "naturally require ... [taking] other's perspectives into consideration" (Bridgeman 1981, p. 1233). The interventions were predominantly led by teachers trained on the protocol (e.g., Chalmers and Townsend 1990; Nakkula and Nikitopoulos 2001; Schonert-Reichl et al. 2015; Silvern et al. 1979). Parents were not involved in the majority of these interventions. The duration of treatment was at least 6 weeks, with a bimodal distribution of 6 or 10 weeks. These interventions frequently included a didactic component $(75 \%)$, typically with the aid of vignettes or videos to illustrate the skill, role-plays $(75 \%)$, discussion in response to vignettes, prompts, and/or role-plays $(67 \%)$, modeling $(50 \%)$, and behavioral rehearsal $(33 \%)$, either by homework assignments or in session activities or games. The use of role plays and filming typically requires participants to engage in multiple or opposing perspectives. Some roleplays and skits were reviewed as a group to further generate discussion or to provide feedback. Some interventions further increased engagement by having participants generate their own role-plays or skits rather than acting out prescribed scenarios (e.g., Chandler et al. 1974).

Another two studies demonstrated partial positive outcomes across populations (Begeer et al. 2011; Castillo et al. 2013). These two interventions were similarly group-based but were led by therapists. The treatment duration was comparable to interventions with successful outcomes, lasting 12 and 16 weeks respectively. Of the common features identified above, these two interventions primarily involved only behavioral rehearsals.

Four studies did not demonstrate positive outcomes on perspective-taking (Marsh et al. 1980; Pecukonis 1990; Schonert-Reichl et al. 2012; Yildiz and Duy 2013). These interventions were all similarly group-based, led by researchers or teachers/staff. Treatment duration was variable, but most did not specify the number of weeks, only the number of sessions, which ranged from 6 to 26 . All of the interventions involved some form of experiential activities, two included role-plays, one included reflective activities, and one did not specify the nature of the experiential activities. Three of these interventions included a didactic component, two included modeling, and two included discussions.

\section{Emotion Recognition}

Across types of populations, six interventions demonstrated positive effects for emotion recognition (Emerson and Holden 1991; Greenbank and Sharon 2013; Lacava et al. 2007; Russo-Ponsaran et al. 2016; Solomon et al. 2004; Stichter et al. 2010). Four of these interventions focused exclusively on emotion recognition (Emerson and Holden 1991; Greenbank and Sharon 2013; Lacava et al. 2007; Russo-Ponsaran et al. 2016). Four of the interventions were group-based, led by teachers or therapists, and two were individual-based (Lacava et al. 2007; RussoPonsaran et al. 2016). Parents were not involved in most of these interventions. Half of these interventions lasted 10-20 sessions (Lacava et al. 2007; Stichter et al. 2010; Solomon et al. 2004). All of these interventions involved modeling of different emotions, by video or live demonstrations, and repeated practice of emotion identification. The majority $(83 \%)$ also included a didactic component, typically with visual or video aids, and half included discussions, such as physiological cues related to an emotion (e.g., Emerson and Holden 1991). 
Another six emotion recognition intervention studies demonstrated partial positive results (Baghdadli et al. 2013; Dadds et al. 2014; Dyck and Denver 2003; Lerner et al. 2011; Silver and Oakes 2001; Soorya et al. 2015). Most of these studies $(66.7 \%)$ focused more broadly on larger social skills training. The majority of these interventions were also group-based, led by teachers or researchers, and one was individual-based. Most of these studies did not include parents as part of the interventions. Treatment duration was somewhat more variable, ranging from 5 to 30 sessions. All of the interventions included some form of experiential learning, such as role plays $(50 \%)$, practice exercises in session (50\%), or homework assignments (33\%). Only $33 \%$ of the interventions included modeling or didactics.

Two interventions did not demonstrate positive outcomes on emotion recognition (Corbett et al. 2011; Dadds et al. 2012). One of the interventions was group-based, and one was individual-based. Both of these interventions were led by therapists. Corbett et al. (2011) targeted socioemotional functioning more broadly and employed musical theater as a strategy. Their intervention varied in intensity depending on the role of the participant in the musical. Dadds et al. (2012) specifically focused on emotion recognition and employed an interactive video program to train emotion identification. Their intervention lasted four sessions. Both of these interventions involved modeling of emotions. Corbett et al. (2011) used role-plays extensively, whereas Dadds et al. (2012) used practice exercises as part of the interventions.

\section{Discussion}

This article reviewed the literature on interventions that targeted emotion recognition and/or perspective-taking among community and clinical samples of adolescents. The review first addressed the efficacy and/or effectiveness of these interventions. Common processes and features across promising and/or effective interventions were identified in the hopes of informing a transdiagnostic approach to targeting these affective abilities in adolescents. In particular, given the pervasive emotion recognition and perspectivetaking deficits among adolescents with callous-unemotional traits and the lack of evidence-based strategies to remediate these affective impairments for this population, results from this article may be particularly informative for refining or developing new treatment strategies for youth with callous-unemotional traits.

\section{Evidence of Efficacy/Effectiveness}

Of the 32 identified studies, 18 involved perspective-taking interventions, 12 involved emotion recognition interventions, and two addressed both skills in the interventions. Overall, $60 \%$ of the studies that targeted perspective-taking demonstrated positive outcomes, and another $10 \%$ demonstrated partial positive outcomes. About a third of these studies involved community samples, and another third focused on youth with AutismSpectrum Disorder or conduct problems.

Evidence of efficacy or effectiveness is more modest for interventions targeting emotion recognition. Overall, $42.9 \%$ of the studies that targeted emotion recognition indicated positive outcomes, and another $42.9 \%$ of the studies indicated partial positive outcomes. These studies are overrepresented by samples of adolescents with autismspectrum disorder $(69.2 \%)$. About $15 \%$ of these interventions involved community samples. None of the studies with promising results for emotion recognition involved samples of youth with conduct problems. Nevertheless, existing interventions that address affective abilities among adolescents seem promising, although we can be more confident about interventions for perspective-taking than emotion recognition.

\section{Common Features Among Effective Interventions}

Interventions for perspective-taking with positive outcomes were commonly implemented by teachers in a group format. The most common duration of interventions was 6 or 10 weeks. The interventions with promising results typically included multiple components including didactic, discussion, rehearsal, modeling, and role-plays. It appears that concurrent inclusion of these components was associated with successful outcomes. Indeed, studies that have mixed results or null results typically involved interventions that included fewer of these elements. The use of real or hypothetical social situations did not appear to differentiate interventions. However, results seem to favor interventions that allowed participants to generate their own scenarios or skits to practice.

Interventions for emotion recognition that have shown positive outcomes were also commonly implemented in a group format, although the leaders varied between therapists and teachers. Interventions that exclusively targeted emotion recognition tended to generate better outcomes compared to interventions that were more diffused (e.g., social skills training). The duration of intervention was typically longer for emotion recognition, commonly ranging between 10 and 20 sessions. Those with successful outcomes tended to involve extensive modeling and didactic training via multiple modalities and repeated practice of emotion identification, either within sessions or between sessions. It is less clear what differentiates interventions that were successful from those that were not aside from the specificity of 
treatment. However, there is some suggestion that inclusion of more elements (i.e., modeling, repeated practice, didactic, discussion, role-plays) may be associated with positive outcomes. The use of real, dynamic, or static stimuli did not appear to differentiate the interventions in terms of efficacy.

\section{Recommendations for Intervention for Youth with Callous-Unemotional Traits}

None of the interventions reviewed targeted emotion recognition or perspective-taking abilities among youth with high levels of callous-unemotional traits. However, based on the promising results and commonalities that emerged among interventions with positive outcomes, several recommendations are offered for interventions for adolescents with callous-unemotional traits to target their affective abilities. Group-based interventions for emotion recognition and perspective-taking appear to be feasible. There is some variability among studies regarding leaders of the interventions, although they are commonly implemented by teachers or therapists/researchers. This approach is consistent with a prevention orientation and implementing services at a systemic level through the school system. Furthermore, group interventions are economical and practical for settings such as the juvenile justice system or residential programs where callousunemotional traits may be more prevalent. There also seems to be a trend in favor of specificity of training. Thus, interventions may benefit by exclusively focusing on emotion recognition or perspective-taking rather than targeting multiple socioemotional skills. Although there does not seem to be a standard duration of treatment, most perspective-taking interventions reviewed last 6 or 10 weeks, and emotion recognition interventions last slightly longer. Across both types of interventions, didactic training, modeling, and rehearsals appear to be essential components. However, more components appear to be associated with more favorable outcomes. The use of real or hypothetical social situations for perspectivetaking and the use of dynamic or static stimuli for emotion recognition do not appear to clearly differentiate interventions in terms of efficacy. Parent involvement does not appear to be necessary for positive outcomes, which is encouraging as it is likely that youth with callous-unemotional traits may be overrepresented in settings where parents are difficult to access, such as incarcerated or residential facilities given their higher propensity for engaging in antisocial and delinquent behaviors. However, peers and trained facilitators in these settings may help exert a positive influence on such youth in the context of group-based interventions to address affective abilities.

\section{Gaps and Future Directions}

Several limitations should be noted regarding the present review. The majority of studies reviewed in this article involved samples of adolescents with Autism Spectrum Disorder and typically-developing adolescents. Few studies involved adolescents with conduct problems or delinquent behaviors. Studies with other populations of adolescents, such as those with attention-deficit/hyperactivity disorder, mood symptoms, global intellectual or learning impairments, schizophrenia spectrum disorders, and brain injuries were scarce or nonexistent, even though research documents emotion recognition and/or perspective-taking impairments associated with these symptoms or disorders (Adams and Markham 1991; Bloom and Heath 2010; Bozorg et al. 2014; Marton et al. 2009; Rocca et al. 2009; Schiffman et al. 2004; Schmidt et al. 2010; van Rijn et al. 2011). The underrepresentation of additional populations limits the present extent of a transdiagnostic approach to targeting such skills among adolescents. Furthermore, early adolescents were overrepresented in the studies included in this review, relative to youth in other age groups. Thus, current findings and conclusions may be most appropriate for this age group (10-13 years) and may not generalize to older adolescents. In addition, the gender composition in these studies does not fully represent the gender ratio typically reported for certain populations. Specifically, females were somewhat overrepresented in studies focusing on conduct and behavioral problems, males were overrepresented in studies focusing on autism spectrum disorder, even accounting for typical gender differences in prevalence rates. Except for studies with typically-developing adolescents, sample sizes were typically small, limiting sufficient power to detect small effects. For example, the average sample size of studies with adolescents with Autism Spectrum Disorder was 25. Although challenging, future treatment outcome studies with clinical samples would benefit from larger sample sizes.

When examining the distribution of skills targeted in the interventions across samples, studies with community samples $(90.9 \%)$ and youth with conduct problems $(80 \%)$ overwhelmingly targeted perspective-taking skills, whereas studies with individuals with autism spectrum disorder (69.2\%) targeted emotion recognition skills. Thus, the differential pattern of results regarding perspective-taking and emotion recognition interventions may be a partial function of the nature of the samples involved in these interventions. Such differences in the types of samples involved in the two types of interventions also render it difficult to draw direct comparisons. Findings from emotion recognition interventions were more tenuous, in light of less consistent findings. Although the majority of studies reviewed included a comparison group, these groups range 
from no treatment/waitlist to placebo to another active treatment. As the body of literature accumulates, it would be beneficial to tease apart the level of efficacy/effectiveness of interventions among varying levels of rigorous designs to obtain a more fine-grained understanding of which interventions work. One would presumably have more confidence in an intervention if it is consistently shown to lead to superior outcomes in perspective-taking or emotion recognition relative to another active intervention that does not target these skills but that controls for other factors (e.g., time and interaction with therapists, positive expectancies).

A file drawer effect may also be present and should be considered whereby treatment outcome studies that focus exclusively on a single skill (i.e., emotion recognition or perspective taking) that yield null or negative findings remain unpublished, whereas treatment outcome studies that focus on multiple skills are published so long as significant findings emerge with some outcome measures. Furthermore, some studies identified in this review did not report sufficient and/or relevant information, such as socioeconomic status and ethnic composition of the sample and statistics to calculate effect sizes, limiting the scope of information and recommendations that can be extracted from the available data. Some studies also did not specify the duration of treatment, which is critical information for future researchers and clinicians wishing to implement the intervention. More precise and comprehensive reporting of data are recommended for future studies. The majority of studies reviewed also did not include a follow-up assessment, raising the question of whether improvements maintain over the long-term.

\section{Conclusion}

Interventions for emotion recognition and perspectivetaking for adolescents appear promising. Some commonalities across interventions for different target populations emerged. Interventions with positive outcomes were typically implemented in a group format by teachers, therapists, or researchers, without parent involvement in the intervention. Specificity in training (i.e., emotion recognition training rather than social skills training) seemed to be associated with more favorable outcomes than interventions targeting multiple socioemotional skills. Core components across interventions included didactic training, group discussion, and behavioral rehearsals. The simultaneous use of multiple components may be associated with more positive outcomes. Such findings can inform researchers and clinicians who are interested in developing or tailoring a program to address these affective abilities in adolescents. One particular population of interest may be adolescents with callousunemotional traits, given that they demonstrate pervasive affective impairments, including deficits in emotion recognition and perspective-taking. The prevailing impression that these youth are treatment-resistant may be in part due to the lack of tailoring of interventions to fit their distinctive emotional processing deficits. These deficits may require more intensive and focused interventions. Therefore, it may be helpful for an intervention for youth with callous-unemotional traits to involve similar aspects identified in the review.

Acknowledgements The authors thank Dr. David Marcus and Dr. Leonard Burns for their feedback on the analysis and presentation of results.

Authors' Contributions JL conceived of the study, conducted the literature search, analyzed and interpreted the data, and drafted the manuscript; CB helped analyze and interpret the data and helped draft the manuscript; CS helped analyze and interpret the data and helped draft the manuscript. All authors read and approved the final manuscript.

\section{Compliance with Ethical Standards}

Conflict of interest The authors declare that they have no conflict of interest.

Ethical Standards APA ethical guidelines were followed in the execution of this research.

\section{References}

Adams, K., \& Markham, R. (1991). Recognition of affective facial expressions by children and adolescents with and without mental retardation. American Journal of Mental Retardation, 96, 21-28.

American Psychiatric Association. (2013). Diagnostic and statistical manual of mental disorders (5th ed.). Arlington, VA: Author.

Anastassiou-Hadjicharalambous, X., \& Warden, D. (2008). Cognitive and affective perspective-taking in conduct-disordered children high and low on callous-unemotional traits. Child and Adolescent Psychiatry and Mental Health, 2, 16. doi:10.1186/17532000-2-16.

Baghdadli, A., Brisot, J., Henry, V., Michelon, C., Soussana, M., Rattaz, C., et al. (2013). Social skills improvement in children with high-functioning autism: A pilot randomized controlled trial. European Child and Adolescent Psychiatry, 22, 433-442. doi:10.1007/s00787-013-0388-8.

Bauminger, N. (2007). Brief report: Individual social-multi-modal intervention for HFASD. Journal of Autism and Developmental Disorders, 37, 1593-1604. doi:10.1007/s10803-006-0245-4.

Begeer, S., Gevers, C., Clifford, P., Verhoeve, M., Kat, K., Hoddenbach, E., et al. (2011). Theory of mind training in children with autism: A randomized controlled trial. Journal of Autism and Developmental Disorders, 41, 997-1006. doi:10. 1007/s10803-010-1121-9.

Blair, R. J. R., Budhani, S., Colledge, E., \& Scott, S. (2005). Deafness to fear in boys with psychopathic tendencies. Journal of Child Psychology and Psychiatry, 46, 327-336. doi:10.1111/j.14697610.2004.00356. 
Blancher-Dixon, J., \& Simeonsson, R. J. (1978). Effect of shared experience on role-taking performance of retarded children. American Journal of Mental Deficiency, 31, 21-28.

Bloom, E., \& Heath, N. (2010). Recognition, expression, and understanding facial expressions of emotion in adolescents with nonverbal and general learning disabilities. Journal of Learning Disabilities, 43, 180-192. doi:10.1177/0022219409345014.

Bozorg, B., Tehrani-Doost, M., Shahrivar, Z., Fata, L., \& Mohamadzadeh, A. (2014). Facial emotion recognition in adolescents with bipolar disorder. Iranian Journal of Psychiatry, 9, 20-24.

Bridgeman, D. L. (1981). Enhanced role taking through cooperative interdependence: A field study. Child Development, 52, 1231-1238.

Bulkeley, R., \& Cramer, D. (1994). Social skills training with young adolescents: Group and individual approaches in a school setting. Journal of Adolescence, 17, 521-531.

Castillo, R., Salguero, J. M., Fernández-Berrocal, P., \& Balluerka, N. (2013). Effects of an emotional intelligence intervention on aggression and empathy among adolescents. Journal of Adolescence, 36, 883-892. doi:10.1016/j.adolescence.2013.07.001.

Chalmers, J. B., \& Townsend, M. A. (1990). The effects of training in social perspective taking on socially maladjusted girls. Child Development, 61, 178-190.

Chandler, M. J. (1973). Egocentrism and antisocial behavior: The assessment and training of social perspective-taking skills. Developmental Psychology, 9, 326-332. doi:10.1037/h0034974.

Chandler, M. J., Greenspan, S., \& Barenboim, C. (1974). Assessment and training of role-taking and referential communication skills in institutionalized emotionally disturbed children. Developmental Psychology, 10, 546-553.

Corbett, B. A., Gunther, J. R., Comins, D., Price, J., Ryan, N., Simon, D., et al. (2011). Brief report: Theatre as therapy for children with autism spectrum disorder. Journal of Autism and Developmental Disorders, 41, 505-511. doi:10.1007/s10803-010-10641.

Dadds, M. R., Cauchi, A. J., Wimalaweera, S., Hawes, D. J., \& Brennan, J. (2012). Outcomes, moderators, and mediators of empathic-emotion recognition training for complex conduct problems in childhood. Psychiatry Research, 199, 201-207. doi:10.1016/j.psychres.2012.04.033.

Dadds, M. R., MacDonald, E., Cauchi, A., Williams, K., Levy, F., \& Brennan, J. (2014). Nasal oxytocin for social deficits in childhood autism: A randomized controlled trial. Journal of Autism and Developmental Disorders, 44, 521-531. doi:10.1007/ s10803-013-1899-3.

Dadds, M. R., Perry, Y., Hawes, D. J., Merz, S., Riddell, A. C., Haines, D. J., et al. (2006). Attention to the eyes and fearrecognition deficits in child psychopathy. The British Journal of Psychiatry, 189, 280-281. doi:10.1192/bjp.bp.105.018150.

Datyner, A., Kimonis, E. R., Hunt, E., \& Armstrong, K. (2016). Using a novel emotional skills module to enhance empathic responding for a child with conduct disorder with limited prosocial emotions. Clinical Case Studies, 15, 35-52. doi:10.1177/ 1534650115588978

Dodd, J. L., Ocampo, A., \& Kennedy, K. S. (2011). Perspective taking through narratives: An intervention for students with ASD. Communication Disorders Quarterly, 33, 23-33. doi:10. $1177 / 1525740110395014$.

Dyck, M. J., \& Denver, E. (2003). Can the emotion recognition ability of deaf children be enhanced? A pilot study. Journal of Deaf Studies and Deaf Education, 8, 348-356. doi:10.1093/deafed/ eng019.

Emerson, P., \& Holden, S. (1991). Teaching children emotional recognition skills. Texas Association for Counseling and Development Journal, 19, 21-26.
Frick, P. J., Ray, J. V., Thornton, L. C., \& Kahn, R. E. (2014). Can callous-unemotional traits enhance the understanding, diagnosis, and treatment of serious conduct problems in children and adolescents? A comprehensive review. Psychological Bulletin, 140, 1-57. doi:10.1037/a0033076.

Greenbank, A., \& Sharon, A. (2013). Recognizing non-verbal social cues promotes social performance in LD adolescents. Alberta Journal of Educational Research, 59, 266-284.

Hawes, D. J., Price, M. J., \& Dadds, M. R. (2014). Callousunemotional traits and the treatment of conduct problems in childhood and adolescence: A comprehensive review. Clinical Child and Family Psychology Review, 17, 248-267. doi:10.1007/ s10567-014-0167-1.

Lacava, P. G., Golan, O., Baron-Cohen, S., \& Myles, B. S. (2007). Using assitive technology to teach emotion recognition to students with Asperger Syndrome: A pilot study. Remedial and Special Education, 28, 174-181.

Lane-Garon, P. S. (1998). Developmental considerations: Encouraging perspective taking in student mediators. Mediation Quarterly, 16, 201-217.

Leist, T., \& Dadds, M. R. (2009). Adolescents' ability to read different emotional faces relates to their history of maltreatment and type of psychopathology. Clinical Child Psychology and Psychiatry, 14, 237-250. doi:10.1177/1359104508100887.

Lerner, M. D., Mikami, A. Y., \& Levine, K. (2011). Socio-dramatic affective-relational intervention for adolescents with Asperger syndrome and high functioning autism: Pilot study. Autism, 15, 21-42. doi:10.1177/1362361309353613.

Lui, J. H. L., Barry, C. T., \& Sacco, D. F. (2016). Callousunemotional traits and empathy deficits: Mediating effects of affective perspective-taking and facial emotion recognition. Cognition and Emotion, 30, 1049-1062. doi:10.1080/ 02699931.2015 .1047327

Marsh, D. T., Serafica, F. C., \& Barenboim, C. (1980). Effect of perspective-taking training on interpersonal problem solving. Child Development, 51, 140-145.

Marton, I., Wiener, J., Rogers, M., Moore, C., \& Tannock, R. (2009). Empathy and social perspective taking in children with attentiondeficit/hyperactivity disorder. Journal of Abnormal Child Psychology, 37, 107-118. doi:10.1007/s10802-008-9262-4.

Muñoz, L. C. (2009). Callous-unemotional traits are related to combined deficits in recognizing afraid faces and body poses. Journal of the American Academy of Child and Adolescent Psychiatry, 48, 554-562. doi:10.1097/CHI.0b013e31819c2419.

Muñoz, L. C., Qualter, P., \& Padgett, G. (2011). Empathy and bullying: Exploring the influence of callous-unemotional traits. Child Psychiatry and Human Development, 42, 183-196. doi:10. 1007/s10578-010-0206-1.

Nakkula, M. J., \& Nikitopoulos, C. E. (2001). Negotiation training and interpersonal development: An exploratory study of early adolescents in Argentina. Adolescence, 36, 1-20.

Pecukonis, E. V. (1990). A cognitive/affective empathy training program as a function of ego development in aggressive adolescent females. Adolescence, 25, 59-76.

Rocca, C. C., Heuvel, E., Caetano, S. C., \& Lafer, B. (2009). Facial emotion recognition in bipolar disorder: A critical review. Revista Brasileira de Psiquiatria, 31, 171-180.

Russo-Ponsaran, N. M., Evans-Smith, B., Johnson, J., Russo, J., \& McKown, C. (2016). Efficacy of a facial emotion training program for children and adolescents with autism spectrum disorders. Journal of Nonverbal Behavior, 40, 13-38. doi:10. 1007/s10919-015-0217-5.

Schiffman, J., Lam, C. W., Jiwatram, T., Ekstrom, M., Sorensen, H., \& Mendick, S. (2004). Perspective-taking deficits in people with schizophrenia spectrum disorders: A prospective investigation. 
Psychological Medicine, 34, 1581-1586. doi:10.1017/ S0033291704002703.

Schmidt, A. T., Hanten, G. R., Li, X., Orsten, K. D., \& Levin, H. S. (2010). Emotion recognition following pediatric traumatic brain injury: Longitudinal analysis of emotional prosody and facial emotion recognition. Neuropsychologia, 48, 2869-2877. doi:10. 1016/j.neuropsychologia.2010.05.029.

Schonert-Reichl, K. A., Oberle, E., Oberlander, T. F., Diamond, A., Lawlor, M. S., Abbott, D., et al. (2015). Enhancing cognitive and social-emotional development through a simple-to-administer mindfulness-based school program for elementary school children: A randomized control trial. Developmental Psychology, 51, 52-66. doi:10.1037/a0038454.

Schonert-Reichl, K. A., Smith, V., Zaidman-Zait, A., \& Hertzman, C. (2012). Promoting children's prosocial behaviors in school: Impact of the "Roots of Empathy" program on the social and emotional competence of school-aged children. School Mental Health, 4, 1-21. doi:10.1007/s12310-011-9064-7.

Silver, M., \& Oakes, P. (2001). Evaluation of a new computer intervention to teach people with autism or Asperger syndrome to recognize and predict emotions in others. Autism, 5, 299-316. doi:10.1177/1362361301005003007.

Silvern, L. E., Waterman, J. M., Sobesky, W. E., \& Ryan, V. L. (1979). Effects of a developmental model of perspective taking training. Child Development, 50, 243-246.

Solomon, M., Goddlin-Jones, B. L., \& Anders, T. F. (2004). A social adjustment enhancement intervention for high functioning autism, Asperger's syndrome, and pervasive developmental disorder NOS. Journal of Autism and Developmental Disorders, 34, 649-668.

Soorya, L. V., Siper, P. M., Beck, T., Soffes, S., Halpern, D., Gorenstein, M., et al. (2015). Randomized comparative trial of a social cognitive skills group for children with autism spectrum disorder. Journal of the American Academy of Child and Adolescent Psychiatry, 54, 208-216. doi:10.1016/j.jaac.2014. 12.005.

Stevens, D., Charman, T., \& Blair, R. J. R. (2001). Recognition of emotion in facial expressions and vocal tones in children with psychopathic tendencies. The Journal of Genetic Psychology, 162, 201-211. doi:10.1080/00221320109597961.

Stichter, J. P., Herzog, M. J., Visovsky, K., Schmidt, C., Randolph, J., Schultz, T., et al. (2010). Social competence intervention for youth with Asperger syndrome and high-functioning autism: An initial investigation. Journal of Autism and Developmental Disorders, 40, 1067-1079. doi:10.1007/s10803-010-0959-1.

U.S. Census Bureau. (2015). 2015 American community survey 1-year estimates. http://factfinder.census.gov/faces/nav/jsf/pages/index. xhtml.

van Rijn, S., Aleman, A., de Sonneville, L., Sprong, M., Ziermans, T., Schothorst, P., et al. (2011). Misattribution of facial expressions of emotion in adolescents at increased risk of psychosis: The role of inhibitory control. Psychological Medicine, 43, 499-508. doi:10.1017/S0033291710000929.

Wölfer, R., Schultze-Krumbholz, A., Zagorscak, P., Jäkel, A., Göbel, K., \& Scheithauer, H. (2014). Prevention 2.0: Targeting cyberbullying @ school. Prevention Science, 15, 879-887. doi:10.1007/s11121-013-0438-y.

Yildiz, M. A., \& Duy, B. (2013). Improving empathy and communication skills of visually impaired early adolescents through a psychoeducation program. Educational Sciences: Theory \& Practice, 13, 1470-1476. doi:10.12738/estp.2013.3.1607. 